A investigação sobre as tendências socioculturais e de consumo como uma nova perspectiva metodológica para a profissão publicitária

María López Vilar

Doutora, Universidad de Murcia / lopezvilarmaria84@gmail.com Orcid: 0000-0003-3864-2810 / lattes

Pedro A. Hellín Ortuño 


\title{
A investigação sobre as tendências socioculturais e de consumo como uma nova perspectiva metodológica para a profissão publicitária
}

\begin{abstract}
RESUMO
Desde a década de 1980, o interesse investigativo e os avanços no campo das tendências socioculturais e de consumo vêm crescendo em nível internacional. Mas, até o momento, não existe nem uma metodologia nem um corpo de técnicas de investigação estabelecidas para tal atividade. É por essa razão que defendemos o estabelecimento e o consenso de uma metodologia juntamente com um conjunto de técnicas investigativas bem estruturadas para 0 desenvolvimento de pesquisas de tendências. Este artigo apresenta a análise de conteúdo e, posteriormente, um estudo comparativo dos métodos e técnicas utilizados até hoje pelos principais observatórios de tendências brasileiras e espanholas. É também um estudo dos setores de consumo na qual tal atividade é desenvolvida e das disciplinas que a servem. Estudo que estamos começando a estender a EE. UU, Reino Unido, França, Itália e Japão. É, sem dúvida, uma contribuição sem precedentes que levará ao estabelecimento da investigação de tendências socioculturais e de consumo, como uma ferramenta essencial dentro da disciplina de comunicação. É uma investigação da comunicação adaptada à complexa rede sociocultural e de consumo que nos rodeia. É também uma ferramenta de investigação, capaz de oferecer soluções inovadoras para marcas contemporâneas e de desenvolver estratégias de comunicação eficientes. Estes juntos serão capazes de atingir efetivamente o consumidor moderno.
\end{abstract}


Palavras-chave: consumo, inovação, marcas, publicidade, estratégias de comunicação, tendências. 


\title{
Research on socio-cultural and consumer trends as a new methodological perspective for the advertising profession
}

\begin{abstract}
Since the 1980s, investigative interest and advances in the field of socio-cultural and consumer trends have been growing internationally. But, so far, there is neither a methodology nor a body of research techniques established for such an activity. It is for this reason that we defend the establishment and consensus of a methodology together with a set of wellstructured investigative techniques for the development of trend research. This article presents the content analysis and, later, a comparative study of the methods and techniques used until today by the main observatories of Brazilian and Spanish trends. It is also a study of the consumer sectors in which this activity is carried out and the disciplines that serve it. I study that we are beginning to extend EE. UU, United Kingdom, France, Italy and Japan. It is undoubtedly an unprecedented contribution that will lead to the establishment of the investigation of socio-cultural and consumer trends, as an essential tool within the discipline of communication. It is an investigation of communication adapted to the complex socio-cultural and consumer network that surrounds us. It is also a research tool, capable of offering innovative solutions for contemporary brands and developing efficient communication strategies. These together will be able to effectively reach the modern consumer.
\end{abstract}

Keywords: consumption, innovation, brands, advertising, communication strategies, trends. 


\title{
Investigación sobre tendencias socioculturales y de consumo como una nueva perspectiva metodológica para la profesión publicitaria
}

\begin{abstract}
RESUMEN
Desde la década de 1980, el interés investigador y los avances en el campo de las tendencias socioculturales y de consumo han ido creciendo a nivel internacional. Pero, hasta el momento, no existe una metodología ni un cuerpo de técnicas de investigación establecidas para tal actividad. Es por ello que defendemos el establecimiento y consenso de una metodología junto con un conjunto de técnicas investigativas bien estructuradas para el desarrollo de la investigación de tendencias. Este artículo presenta el análisis de contenido y, posteriormente, un estudio comparativo de los métodos y técnicas utilizados hasta hoy por los principales observatorios de las tendencias brasileñas y españolas. También es un estudio de los sectores de consumo en los que se desarrolla dicha actividad y las disciplinas que la atienden. Estudio que estamos empezando a extender EE. UU, Reino Unido, Francia, Italia y Japón, es sin duda una aportación inédita que conducirá al establecimiento de la investigación de las tendencias socioculturales y de consumo, como herramienta imprescindible dentro de la disciplina de la comunicación. Se trata de una investigación de la comunicación adaptada a la compleja red sociocultural y de consumo que nos rodea. También es una herramienta de investigación, capaz de ofrecer soluciones innovadoras para marcas contemporáneas y desarrollar estrategias de comunicación eficientes. Estos juntos podrán llegar de manera efectiva al consumidor moderno.
\end{abstract}

Palabras clave: consumo, innovación, marcas, publicidad, estrategias de comunicación, tendencias. 


\section{INTRODUÇÃO E HIPÓTESE DESTE ESTUDO}

A investigação das Tendências Socioculturais e de Consumo continua a se estender diariamente ao campo das comunicações, e desde os anos 1980, diferentes empresas americanas, latino-americanas e europeias estão trabalhando para o desenvolvimento e aperfeiçoamento deste tipo de investigação; estimulando o conhecimento nas marcas, produtos, serviços e instituições governamentais com uma ferramenta de comunicação competitiva capaz de possibilitar uma visão eficiente do consumidor atual. Os resultados obtidos por meio desse tipo de investigação são oferecidos como instrumento de inovação e vantagem competitiva; diante de um mercado exponencialmente saturado e sujeito a constantes mudanças, no contexto de um consumidor progressivamente mais complexo, e de gostos e preferências flutuantes. Mas; dada a sua breve experiência a nível nacional, tanto quanto a nível internacional como as diferentes estratégias empresariais que prevalecem em cada país, a investigação das Tendências Socioculturais e de Consumo não conta hoje com um método estruturado para o seu desenvolvimento e aplicação, nem em um conjunto de técnicas de investigação estabelecidas. Consequentemente, e partindo dessa hipótese, este artigo tem como objetivo apresentar um estudo comparativo de diferentes métodos e técnicas utilizadas para o desenvolvimento da investigação de Tendências Socioculturais e de Consumo e, de forma semelhante, para se fazer conhecer as diferentes esferas de consumo em que tal atividade é desenvolvida e quais disciplinas são atualmente utilizadas no desenvolvimento deste tipo de investigação.

Para desenvolver este estudo comparativo, o foco tem sido a análise de negócios dedicados à investigação das 
Tendências Socioculturais e de Consumo na Espanha e no Brasil, com a intenção de estabelecer métodos e técnicas para o desenvolvimento deste tipo de investigação. Em um futuro próximo, pretende-se estender o estudo comparativo a outros países, como EUA, Reino Unido, França, Itália e Japão, com o objetivo de estabelecer um mapa de métodos e técnicas consolidados para a investigação de tendências.

A escolha do Brasil foi feita no início dessa conscientização, numa época em que o país estava proliferando significativamente sites especializados, blogs e reportagens sobre tendências, coolhunters, agências de coolhunting e abertura de subsidiárias internacionais de negócios no Brasil; como o WGSN (World Global Style Network). Tudo isso produziu prova da propagação e do maior grau de legitimidade desse tipo de atividade profissional no Brasil. Outro forte indicador vem do interesse acadêmico no Brasil por meio de cursos, monografias e projetos que tratam da investigação e aplicação de tendências em setores específicos.

\section{TENDÊNCIAS SOCIOCULTURAIS E DE CONSUMO}

Como Semprini (2006) observou, até o final da década de 1970 a compreensão do consumo girava em torno do consumidor e, consequentemente, e em termos de segmentação do mercado, foi realizada com base em categorias sócio demográficas (classe social, ganhos, idade, etc.). Nos anos 1980, ficou aparente que esses indicadores não eram suficientes para explicar os padrões de comportamento determináveis nos consumidores, nem para as mudanças sociais e culturais definíveis que afetavam seu comportamento. Foi então que surgiu a necessidade de reformulação de estratégias tradicionais de marketing e comunicação com o objetivo de estabelecer novas 
metodologias que pudessem contribuir para a compreensão do comportamento do consumidor contemporâneo, e que pudessem se desenvolver de acordo com uma nova complexidade social e as mudanças frenéticas na sociedade atual.

Além disso, outros teóricos, como Pérez, Trindade, Hellín e Lencaster (2012), argumentam que os estudos de mercado, baseados em conceitos tradicionais de classe alvo e social, não respondem pela complexa rede de links significativos estabelecidos entre pessoas e suas marcas. Concordando com esses teóricos, o analista de tendências socioculturais estabelece novas oportunidades para a investigação do mercado, marketing e publicidade. O analista de tendências desperta a consciência de quais são as motivações que desencadeiam comportamentos no campo do consumo; revelando movimentos mais profundos ancorados no contexto sociocultural e, a partir deles, novos cenários e oportunidades são propostos para a inovação. O Analista de Tendências Socioculturais percebe sinais e valores emergentes que, necessariamente, não estão ligados a padrões demográficos, de gênero, renda ou classe social.

O termo tendência é atualmente usado de forma diferente em diferentes profissões e contextos: estatístico, econômico, histórico, etc. e, em todos esses, o conceito de mudança está sempre presente. No entanto, dado o nosso campo de pesquisa, a definição de uma tendência que nos preocupa é a definição de uma tendência do ponto de vista das disciplinas das ciências sociais.

A palavra tendência é definida no Corpus Canónico de literatura inglesa, o Oxford English Dictionary, como "a direção em que algo está mudando ou algo está sendo desenvolvido; mudanças ou desenvolvimentos em uma direção específica". ${ }^{2}$ 
O pesquisador especialista em tendências sociais, Vejlgaard (2008: 8), indica que: "Para um sociólogo das tendências, não é algo que já aconteceu; é uma predição de algo que ocorrerá em um determinado momento". Acrescenta, além disso, que "antes que uma tendência ocorra dentro do ambiente social e do consumo que rivaliza com o nosso, existe um processo social anterior que chama a atenção de todos e, por isso, não é tratado como um súbito aparecimento. Tal processo precisa de tempo para uma tendência chegar à população de um país e provocar mudanças. Uma das chaves para detectar uma tendência", de acordo com Henrik Vejlgaard (2008), é ver que um estilo similar, tanto em o contexto social referido como no consumo, torna-se evidente em diferentes categorias de hábitosii ou produtos, permitindo-lhe revelar-se como uma tendência emergente e não apenas uma moda passageira ou momentânea.

Para Córdoba-Mendiola (2009) pai fundador e diretor de The Hunter, Barcelona (Espanha), as tendências traçam uma direção e uma intenção e, como tal, são usadas pelos meios de comunicação e setores profissionais para explicar as tendências do comportamento do consumidor. É necessário analisá-los separadamente e entender que alguns são fontes de previsão e outros são o resultado de expressões já realizadas.

Caldas (2004), sociólogo brasileiro, pesquisador de tendências e fundador do Observatório de Sinais, ressalta que a palavra tendência reflete um tipo de sensibilidade específica para a qual a modernidade abriu o caminho e a pósmodernidade acabou por santificá-la. A palavra tendência agora vem carregada de significados de alteridade, na medida em que ela só pode existir em sua função de atração de movimento ou expansão, exercida por outro objeto. 
Mark J. Penn (2008), um consultor dos Estados Unidos em comunicação política, defende a ideia de que as forças mais poderosas da sociedade são tendências emergentes e contra intuitivas; aquelas que estão moldando o futuro diante de nossos olhos e que o mesmo autor define como microtendências; pequenas forças imperceptíveis que podem envolver até $1 \%$ da população mundial, e que moldam nossa sociedade de maneira irreversível. E para que possamos realmente entender o que está acontecendo, devemos disponibilizar os melhores métodos de investigação.

Para Pérez e Solana (2006) "As tendências se mostram como espaços em que os valores e atitudes predominantes se refletem como resultado de mudanças que ocorrem na sociedade; aquelas que são interpretadas de maneira diferente por diferentes grupos de consumidores" (p. 136).

Como William Higham (2009) observa, talvez devido à sua história breve e multifacetada, o processo de análise de tendências ainda tem que ganhar um nome concordado; na medida em que as tendências se tornaram importantes, nomes diferentes são usados para descrever o processo de analisá-las. Um termo inicial foi o da "futurologia". Mais tarde, várias corporações que introduziram estudos de tendências cotidianas usaram o termo "coolhunter". Atualmente, os termos mais comuns e usados mundialmente são "tendências" (trends) e "trend-spotting", "previsão de tendências" (trend forecasting) ou "previsão de tendências" (trend prediction). Por isso, ainda hoje, torna-se difícil formular com precisão uma definição de tendências socioculturais e de consumo; mesmo para um analista de tendências.

Com tudo isso, é agradável fechar esse ponto fornecendo nossa própria definição de tendências socioculturais e de consumo. 
As tendências socioculturais e de consumo são indicadores que emergem do ambiente que nos rodeia e que tendem a prever mudanças nas esferas e no consumo cultural e no próprio comportamento do consumidor. Essas tendências socioculturais e de consumo são uma lente, através da qual, o contexto social e de consumo que nos rodeia, pode ser observado.

\section{METODOLOGIA E DESIGN DA PESQUISA}

\subsection{Análise preliminar}

Para alcançar uma abordagem ao nosso estudo, o primeiro passo foi proceder à tarefa de localizar negócios dedicados à investigação de tendências socioculturais e de consumo no Brasil e na Espanha, para posterior análise de conteúdo e comparações relacionadas. Para estes últimos propósitos, o desenvolvimento de uma meticulosa metodologia de filtragem e gestão foi realizada.

\section{A) Seleção de Mídia para Realização das Pesquisas}

As fontes documentais que se pode acessar para conhecer o estado da Trend Investigation, bem como a aplicação dos resultados provenientes das referidas investigações no setor publicitário, são escassas. Monografias específicas sobre a investigação de Tendências Socioculturais e de Consumo dificilmente existem. Também não há artigos científicos que entrem no escopo deste tipo de investigação. Consequentemente, o único meio que foi disponibilizado para localizar empresas dedicadas à pesquisa atual foi o WebSearch-Engine. Para esta investigação, em particular, os três Web-Search-Engines mais usados foram selecionados. Estes, de acordo com os estudos da Statista, são o Google em 
primeiro lugar, seguido pelo Bing e depois pelo Yahoo. Decidiu-se estabelecer mais de um mecanismo de busca, ampliando assim o leque de possibilidades na busca de empresas dedicadas à investigação de tendências.

\section{B) Termos estabelecidos para o Search Launch}

Diferentes termos de pesquisa foram determinados para 0 lançamento da Pesquisa; sempre levando em conta a língua do país de origem (português e espanhol). Os termos de pesquisa estabelecidos foram os seguintes:

- Observatório de Tendências

- Investigação de tendência

- Investigação de tendência de consumo

- Blog de tendência

- Blog de tendência de consumo

- Coolhunting

- Coolhunter

Um total de sete termos investigativos foram estabelecidos e cada um foi configurado em cada um dos três mecanismos de busca em cada um dos dois países, e a análise preliminar foi aplicada. Aceito que, embora existam sete termos investigativos, estes são vistos como duplicados; dado que a investigação abrange dois países.

Uma vez que os sete termos investigativos foram estabelecidos e ordenados em cada um dos três buscadores de empresas de investigação de tendências, em cada um dos dois países, foi decidido restringir o exercício a 8.400 sites / páginas para o exame preliminar, por exemplo. Em cada termo investigativo, os primeiros 200 resultados fornecidos por cada um dos três mecanismos de busca em cada um dos dois países seriam usados em uma filtragem adicional. 0 
resultado de 600 sites para cada um dos sete termos investigativos em cada país avança para 4.200 sites para cada país e, portanto, para 8.400 para os dois países juntos.

\section{C) Critérios de Filtragem para Portais Selecionados}

Dois critérios de filtragem foram estabelecidos para trabalhar na amostra preliminar bruta de 8.400 sites / páginas.

Em primeiro lugar, que o termo investigativo utilizado na pesquisa estaria presente em pelo menos um dos três campos dos sites / páginas recuperadas: os títulos das páginas, sua descrição ou, em sua URL.

Durante este processo de filtragem, duplicatas foram eliminadas simultaneamente ${ }^{3}$.

O resultado foi o seguinte:

- Brasil Observatório de Tendências da Espanha

- Trend Observatory

- Trend Investigation

- Trend Investigation

- Consumo Trend Investigation

- Consumption Trend Investigation

- Trend Blog

- Tendência Blog

- Consumo Tendência Blog

- Consumo Tendência Blog

- Coolhunting

- Coolhunting

- Coolhunter

- Coolhunter

Como segundo critério de filtração, cada uma dessas empresas, observatórios ou consultorias que tratam de 
tendências selecionadas (66 no Brasil, e 75 na Espanha) devem cumprir com três critérios de maneira simultânea. A presença desses três critérios foi condição sine qua non e, em primeira instância, para as empresas selecionadas passarem ou não, da amostra bruta para a amostra final do estudo.

\section{Investigação Contínua ao longo do tempo.}

Como foi indicado quando se definiram as Tendências Socioculturais e de Consumo, surgem indicações cotidianas em diferentes esferas de consumo; indicações que preveem mudanças. Por essa razão, a investigação permanente resulta em um requisito fundamental da Trend Investigation.

\section{Presença de uma Metodologia ou Metodologias de} Investigação.

A presença de uma Metodologia, ou Metodologias, de Investigação, resulta em um critério fundamental de conformidade por parte de empresas que atendem ao requisito final do estudo: conhecer quais são os métodos e técnicas investigativas atualmente utilizados na investigação de tendências; tanto a nível nacional como internacional.

III Presença em Investigações de Tendências de Diferentes Setores de Atividade.

As tendências socioculturais e de consumo surgem em formas simultâneas em diferentes esferas de atividade e podem ser aplicadas - estrategicamente - em diferentes setores de consumo.

Uma vez que todos esses filtros são aplicados a todas as empresas selecionadas na primeira instância (de dados brutos), um total de 10 empresas (5 do Brasil e 5 da Espanha), finalmente passam a fazer parte da amostra final da investigação. 


\section{ANÁLISE DE CONTEÚDO E RESULTADOS DA COMPARAÇÃO}

Por fim, e em busca do objetivo da investigação, foi realizada uma análise do conteúdo da amostra final e, quando obtida, uma análise comparativa de conteúdo posterior foi para esclarecer a hipótese do trabalho. Cada uma das empresas que se conformavam com a amostra foi estudada; assim como suas metodologias ou métodos de investigação, as diferentes técnicas utilizadas, os vários setores de consumo onde esses métodos e técnicas foram aplicados e as disciplinas - dentro do grupo de investigação - nas quais cada uma das empresas dependia.

Tanto nos brasileiros, como nos espanhóis, os negócios de investigação de tendências, desenvolveram uma investigação contínua ao longo do tempo e praticaram o monitoramento de Indicadores em diferentes esferas de consumo com o objetivo de assim detectar tendências. Três dos negócios brasileiros de investigação de tendências - em contraste com os espanhóis - desenvolveram programas específicos para detecção de tendências: WGSN (Mapa de Percepção do Cliente), ODES (Mapa de Valores) e Inova Consulting (Zeitgeist Trend Model). $\mathrm{Na}$ Espanha, essas iniciativas não foram encontradas; nas empresas espanholas, só se utiliza a investigação contínua no tempo e o monitoramento de áreas precisamente nomeadas.

Nos dois países, as empresas usam uma ampla gama de técnicas com o objetivo de detectar tendências. Ocupando as três posições principais são as técnicas de pesquisa de escritório, (utilizado por 9 dos 10 observatórios participantes que estejam em conformidade com a amostra), a observação participante (utilizado por 8), e a técnica Delphi e 
Coolhunting, usado por 7 dos 10 observatórios ${ }^{4}$.

Dentre as técnicas mais utilizadas pelas empresas brasileiras de pesquisa, destacam-se as pesquisas Desk Research, Participant Observation e Delphi. Estes são seguidos de perto pelas técnicas proprietárias do Coolhunter e por Entrevistas em Profundidade. E entre as técnicas mais utilizadas pelas empresas de investigação espanhola, destacam-se as técnicas proprietárias de Coolhunter, Desk Research, Ethnography e Participant Observation.

Vale ressaltar que as empresas brasileiras trabalham simultaneamente em uma variedade muito maior de esferas do que as empresas espanholas. É isso que lhes permite contar com uma gama mais ampla de possibilidades, a partir das quais os sinais emergentes são detectados. Enquanto empresas espanholas trabalham em 9 setores, as empresas brasileiras têm até 20 setores diferentes presentes no desenvolvimento de pesquisas de tendências.

A presença de uma grande variedade de disciplinas profissionais é notável tanto no Brasil quanto nos Negócios Espanhóis de Investigação. Comunicação, Marketing, Sociologia, Design, Antropologia e Semiótica, são as principais disciplinas presentes nos negócios brasileiros. Enquanto Comunicação, Marketing, Design, Antropologia e Sociologia são os que mais se destacam entre os empresários espanhóis, a presença da Semiótica nas equipes de investigação dos negócios brasileiros é interessante. Como as tendências são antecipáveis por meio de sinais emitidos por diversas esferas sociais e de consumo, acredita-se que a Semiótica, como teoria que estuda os sinais dentro da vida social, torna-se de grande importância para a investigação de Tendências Socioculturais e de Consumo.

Como Floch (1990) declarou, a Semiótica é, acima de tudo, uma relação específica com significado; um foco direcionado 
para tudo que tenha significado; Objetos de significado são as únicas realidades com as quais a semiótica está envolvida e quer se preocupar.

Pode ser tratado como um texto, um logotipo, um filme, um comportamento ou qualquer tipo de manifestação. Nos estudos de Floch (1990), pode-se ver como o mundo do significado é inteligível para o semiótico cujo objetivo é, e continuará sendo, a descrição das condições da produção e a compreensão do significado. O semiótico, além disso, investiga o sistema de relações que formam as invariáveis produções e compreensões de conjuntos, a partir de uma análise das variáveis que são os signos.

\section{CONCLUSÃO}

O desenvolvimento deste trabalho de pesquisa possibilitou a corroboração da hipótese de linha de base. Até hoje não existe uma metodologia acordada nem um conjunto de técnicas investigativas estabelecidas para a Investigação Sociocultural e de Tendências de Consumo. Isso apesar do crescimento de alta velocidade dessa atividade. A falta de um agrupamento de métodos e técnicas unificadas para lidar com a investigação de tendências é muito aparente. A inovação estratégica baseada na aplicação de tendências é uma prática que não traz nada além de uma vantagem competitiva para as marcas.

O Brasil e a Espanha realizam investigação de tendências sobre dois pilares principais: Investigação Contínua ao longo do tempo e Monitoramento de Indicações em diferentes Setores de Atividade. Pode-se deduzir que, quando se fala da prática de detecção de tendências, se fala de uma prática que exige uma investigação contínua em uma dimensão espaçotemporal. O monitoramento de tendências é feito de forma 
paralela, tanto no Brasil quanto na Espanha, em diversas esferas de consumo. A partir disso, podemos extrair uma máxima. Quanto mais uma atividade é permanentemente e continuamente monitorada por um período por empresas de investigação; maior será a possibilidade de detectar sinais emergentes que, juntos, revelarão tendências.

A investigação de tendências no Brasil baseia-se em um maior número de métodos e técnicas de investigação e, além disso, na aplicação de investigações sobre um maior número de setores de consumo. Tudo isso indica um maior conhecimento sobre o mainstreaming e o pragmatismov que envolvem tendências de consumo. Mostram também uma maior consciência da importância da investigação de tendências como uma nova ferramenta de comunicação estratégica adaptada a novos tempos.

Dado que as tendências de consumo são o resultado da interpretação de sinais detectados em diferentes esferas sociais e de consumo, defendemos a coordenação interdisciplinar como base apropriada de toda abordagem investigativa que queira entrar no estudo das tendências de consumo. Todas e cada uma das esferas sociais e de consumo (da publicidade ao varejo, da educação à política, dos alimentos ao esporte, etc.) fornecem sinais emergentes que, quando ligados a metodologias e técnicas apropriadas para sua detecção e interpretação, podem ser traduzidos em valores que emergem na forma de tendências.

A investigação das tendências de consumo gera uma grande quantidade de informações sobre as diferentes esferas sociais e de consumo que nos cercam. O poder depende, em primeiro lugar, do conhecimento, o que as tendências fornecem, tanto atuais quanto futuras, e então pressupõe uma grande vantagem para todas as marcas, produtos ou serviços que querem avançar com inovações competitivas, 
para se tornar a preferência dos consumidores contemporâneos antes que tais preferências mudem.

As Tendências Socioculturais e de Consumo se apresentam como uma fonte de geração de conhecimento que, quando aplicada de maneira estratégica, permitem o desenvolvimento de soluções inovadoras para marcas, produtos e serviços. Mas ainda resta o desenvolvimento de um corpo metodológico de conhecimentos e de técnicas investigativas bem estruturadas, para que este potencial inovador seja progredido e corretamente aplicado.

Espera-se que as ciências sociais, e particularmente a comunicação e a publicidade, se comprometam com isso.

\section{Notas}

${ }^{1} \mathrm{~A}$ palavra "tendencia" em espanhol carrega duas dimensões do tempo; tanto a inclinação de curto prazo como a de médio e longo prazo, mais maduras, mais mensuráveis, enquanto a "tendência" em inglês está mais relacionada com a anterior. Além disso, a palavra "tendência" em inglês incorpora necessariamente seus primórdios na "tendência" de curto prazo, mas vai além dela para o médio e longo prazo.

2Para a compreensão dessa abordagem, e como defende Henrik Vejlgaard (2008), entendemos por hábito uma forma de comportamento não reflexivo, autossustentável e autônomo que surge como resultado de séries repetitivas. 0 hábito substitui, de certa forma, o processo racional. Isto é, o hábito se torna o impulso determinante da escolha supostamente fundamentada.

${ }^{3} \mathrm{O}$ lançamento das pesquisas usando os diferentes Termos estabelecidos e os diferentes mecanismos de pesquisa selecionados resultou na eliminação de duplicatas, pois havia portais que foram recuperados em diferentes ocasiões. Tudo 
isso deu origem ao fato de que o número de portais finalmente selecionados foi drasticamente reduzido.

${ }^{4}$ As empresas dedicadas à investigação de tendências nem sempre são definidas da mesma maneira. Portanto, é possível encontrar: Tendências Consultorias, Tendências Investigações, Tendências Observatórios, etc.

${ }^{5}$ Tendências são pragmáticas. A vigilância permanente das tendências (seu monitoramento contínuo) e sua aplicação estratégica (para identificação de oportunidades de negócios, abordagens e desenvolvimento de produtos e serviços, gestão eficiente de marcas ou criação de processos de inovação) devem ser realizadas simultaneamente. Sem o efeito de equilíbrio da aplicabilidade, a identificação de tendência perde seu significado.

\section{Referências}

BOX 1824. Disponível em: http://www.box1824.com.br. Acesso em: 10 jul. 2015.

CALDAS, Dario. Observatório de Sinais: Teoria e Prática da Pesquisa de Tendências. São Paulo: Senac, 2014.

CÓRDOBA-MENDIOLA, Daniel. Coolhunting: Cazar y gestionar as tendencias que mueven el mundo. Barcelona: Gestión 2000, 2009.

COSKUNER-BALLI, Gokcen; SANDIKCI, Özlem. A aura de novos produtos : como os consumidores mediam a novidade. Journal of Consumer Behavior, 2014, 13 (2), 122-130.

FLOCH, Jean-Marie. Sémiotique, marketing e comunicação. Sous les signes, les stratégies. París: Presses Universitaires de France. 1990.

HIGHMAN, William. A próxima grande coisa: identificar e prever tendências de consumo para lucro. 2009. Reino Unido: Página de Kogan.

INOVA CONSULTING. Disponível em:

http://www.inovaconsulting.com.br. Acesso em: 10 jul. 2016.

KUO-experiência. Disponível em: http://www.kuo-experience.es. Acesso em: 20 ago. 2016.

LA ROCHA. Disponível em: http://www.larock.marketing. Acesso em:10 ago. 2016.

NETHUNTING. Disponível em: http://www.nethunting.es. Acesso em: 17 ago. 2016. 
OBSERVATORIO DE SINAIS. Disponível em:

http://observatoriodesinais.com.br. Acesso em: 10 jul. 2016.

OBSERVATORIO DE TENDENCIAS DEL HÁBITAT. Disponível em: http: // www.tendenciashabitat.es. Acesso em: 10 ago. 2016.

PENN, Mark J. Microtrends: as pequenas forças por trás das grandes mudanças de amanhã. Nova York: Hachette Book Group. 2007.

PEREZ, C. et al. Alvo y tendencias de consumo en la contemporaneidad: Perspectivas teóricas para entender nuevos sentidos. In: Anais do $\mathbf{1 0}^{\circ}$ Congresso Mundial da Associação Internacional de Estudos Semióticos (IASS/AIS), Universidade da Corunha, España, pp. 1077-1086, 2012.

PÉREZ, P.; SOLANAS, I. Incidencia de los estilos de vida en la publicidad y el marketing. Trípodos, (18), 123-138, 2006.

SEMPRINI, Andrea. A Marca Pós-Moderna: Poder e Fragilidade da Marca na Sociedade Contemporânea. São Paulo: Estação das Letras, 2006.

SURGENIA. Disponível em: http://www.surgenia.es. Acesso em: 30 ago. 2016.

LABORATÓRIO DE TENDÊNCIA BARCELONA. Disponível em: http://www.trendslabbarcelona.com. Acesso em: 30 ago. 2016.

V AN STEET, E.; SPEA, N. O.; FABRIZE, R. Ponto de compra ou ponto de frustração? Tendências de frustração do consumidor e resposta em um cenário de varejo. Journal of

ConsumerBehavior, 12 (5), 389-400, 2013.

VEJLGAARD, Henrik. Anatomia de uma tendência. Nova York: McGraw-Hill, 2013.

Rede Global de Estilo Mundial. Disponível em: www.wgsn.com. Acesso em: 21 jul. 2016. 